\title{
Disconnections kinks and competing modes in shear-coupled grain boundary migration
}

\author{
N. Combe, ${ }^{*}$ F. Mompiou, and M. Legros \\ Centre d'Elaboration de Matériaux et d'Etudes Structurales, CNRS UPR 8011, 29 rue J. Marvig, BP 94347, 31055 Toulouse cedex 4, France \\ and Université de Toulouse, UPS, F-31055 Toulouse, France
}

(Received 18 September 2015; revised manuscript received 16 December 2015; published 15 January 2016)

\begin{abstract}
The response of small-grained metals to mechanical stress is investigated by a theoretical study of the elementary mechanisms occurring during the shear-coupled migration of grain boundaries (GB). Investigating a model $\Sigma 17(410)$ GB in a copper bicrystal, both $\langle 110\rangle$ and $\langle 100\rangle$ GB migration modes are studied focusing on both the structural and energetic characteristics. The minimum energy paths of these shear-coupled GB migrations are computed using the nudge elastic band method. For both modes, the GB migration occurs through the nucleation and motion of disconnections. However, the atomic mechanisms of both modes qualitatively differ: While the $\langle 110\rangle$ mode presents no metastable state, the $\langle 100\rangle$ mode shows multiple metastable states, some of them evidencing some kinks along the disconnection lines. Disconnection kinks nucleation and motion activation energies are evaluated. Besides, the activation energies of the $\langle 100\rangle$ mode are smaller than those of the $\langle 110\rangle$ one except for very high stresses. These results significantly improve our knowledge of the GB migration mechanisms and the conditions under which they occur.
\end{abstract}

DOI: 10.1103/PhysRevB.93.024109

\section{INTRODUCTION}

The plastic properties of metals are usually driven by the mobility of dislocations. However, recent experiments have shown that under specific conditions (dislocation depletion, nanocrystalline materials, bicrystal...), grain boundaries (GBs) can themselves participate in plastic deformation [1-5]. Among the possible GB-based mechanisms, the shearcoupled GB migration is especially efficient compared to other mechanisms [6]: The GB migration over a distance $m$ is accompanied by the relative in-plane translation $d$ of the two grains forming the GB. The coupling factor $\beta=\frac{d}{m}$ characterizes this migration. Despite experimental investigations [7-9] and recent theoretical studies [10-16], the details of the shear-coupled migration mechanisms remain poorly known.

Analytical models for coherent tilt boundaries provide quantitative values for the coupling factor $\beta$ as a function of the misorientation angle $\theta$ of the GB $[17,18]$. Especially, [001] symmetric tilt GBs in FCC crystals can shear couple along two modes, the $\langle 100\rangle$ and $\langle 110\rangle$ modes, having, respectively, a positive and a negative coupling factor. These two modes, observed experimentally [7], have been generally studied by molecular dynamics (MD) simulations performed on $\mathrm{Cu}$ bicrystals at different temperatures: Whereas high-angle GB $\left(\theta \gtrsim 35^{\circ}\right)$ migrate following the $\langle 110\rangle$ mode whatever the temperature, low-angle $\mathrm{GB}\left(\theta \lesssim 35^{\circ}\right)$, respectively, migrate under the $\langle 100\rangle$ and $\langle 110\rangle$ modes at high and low temperatures $[10,19]$.

This paper aims at understanding how small-grained metals respond to mechanical stress and more precisely at clarifying the change in the shear coupling mode of a low-angle GB as a function of temperature. We study the competition between the $\langle 100\rangle$ and $\langle 110\rangle$ modes, examining their structural and energetic characteristics. The conditions under which one mechanism is favored over the other are precised. Beyond

*nicolas.combe@cemes.fr that, this paper evidences not only the role of disconnection in the GB migration but also the existence and the role of kinks along disconnections.

Section II describes the system under study and the simulations methods. Section III investigates the elementary mechanisms of the $\langle 100\rangle$ and $\langle 110\rangle$ GB migration modes. Finally, in Sec. IV, comparing the activation energies for both GB migration modes, the conditions under which one mechanism is favored over the other are detailed. Section V concludes this paper.

\section{SIMULATION METHOD}

A comparative study of low-angle GBs with different orientation angles and in different materials is beyond our current computing possibilies. The shear coupled migration of the $\Sigma 17(410)$ GB is investigated in a Cu bicrystal model using the MD simulation package LAMMPS [20] with an embeddedatom method potential [21]. The low-angle $\Sigma 17(410)$ GB has been chosen because it migrates following the $\langle 100\rangle$ and $\langle 110\rangle$ modes at high and low temperature, respectively [10]. The simulation cell displayed in Fig. 1(a) contains two symmetric grains of a perfect FCC copper crystal disorientated relative to each other by a $28.07^{\circ}$ angle around the [001] direction. The formation of a symmetric coincident site lattice (CSL) tilt boundary $\Sigma 17(410)$ results at the interface. The equilibrium configuration of this GB already reported in the literature [10] is shown in Fig. 1(b) and displays characteristic equilibrium structural units. Periodic boundary conditions are applied in the [140] ( $y$ axis) and [001] ( $z$ axis) directions. Throughout the paper, crystal axes are given using the grain 1 lattice orientation. The simulation cell $x$ ( $x$ direction along [410]), $y$, and $z$ sizes are, respectively, $10.7 \mathrm{~nm}, 11.8 \mathrm{~nm}$, and $1.4 \mathrm{~nm}$, and the cell contains 15680 atoms. The characteristics of larger cells used for the study of disconnection kinks in Sec. III B will be given later. The atoms of two 1.6-nm-thick slabs at the top and bottom of the cell [see Fig. 1(a)] have relative positions frozen to the perfect lattice ones: The relative shear displacement (SD) $d$ of these slabs in the $y$ direction imposes 


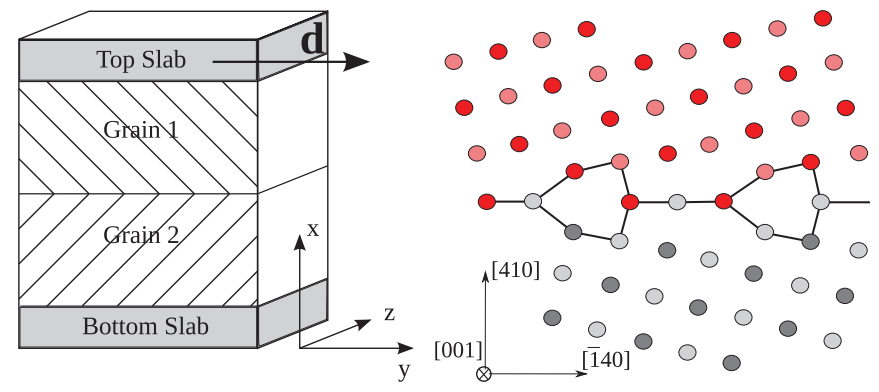

FIG. 1. (a) Sketch of the simulation cell. (b) Equilibrium structure of the $\Sigma 17$ (410) GB projected along the [001] direction: red (salmon) and black (gray) atoms belong to grain 1 and 2. Red (black) and salmon (gray) atoms belong to (001) planes spaced by half the lattice parameter. Solid lines, a guide to the eyes, display the GB position and the structural units. For black and white printing, red, gray, and pink atoms appear as dark gray, gray, and light gray atoms.

a shear stress on the GB. Shear stresses are calculated using the virial stress tensor [22].

In order to study and compare the $\langle 110\rangle$ and $\langle 100\rangle$ modes, sets of initial $i(d)$ and final $f^{\langle 110\rangle}(d)$ and $f^{\langle 100\rangle}(d)$ atomic configurations (i.e., before and after the GB migration) are produced for various applied SD $d$. The initial state of the GB $i(d=0)$, the same for both modes, is provided by the equilibrium GB structure [Fig. 1(b)] and is thus free of any shear stress. The final configurations $f^{\langle 110\rangle}(d=0)$ and $f^{\langle 100\rangle}(d=0)$ after the migration under the $\langle 110\rangle$ and $\langle 100\rangle$ modes are, respectively, produced by shearing the bicrystal at $400 \mathrm{~K}$ and at $0 \mathrm{~K}$. For both modes, after the GB migration, the slabs are moved back at $0 \mathrm{~K}$ to the position $d=0$. Shearing of the GB at $400 \mathrm{~K}$ is performed by MD simulations in the NVT ensemble using a Noose-Hoover thermostat with shearing velocities of $9.4 \times 10^{-3} \mathrm{~m} \mathrm{~s}^{-1}$ and is followed by a quench to $0 \mathrm{~K}$ just after the GB migration. Shearing at $0 \mathrm{~K}$ results from the increase of the SD $d$ by increments of $6 \mathrm{pm}$ : The potential energy is minimized at each step using a conjugate gradient algorithm. The sets of initial and final configurations $i(d), f^{\langle 110\rangle}(d)$, and $f^{\langle 100\rangle}(d)$ of the GB for various applied SD $d$ are produced by an elastic shear of the configurations $i(0)$, $f^{\langle 110\rangle}(0)$, and $f^{\langle 100\rangle}(0)$ at $0 \mathrm{~K}$.

Figures 2(a) and 2(b) report the shear stress and the potential energy of the bicrystal as a function of the SD $d$ for these configuration sets calculated at $0 \mathrm{~K}$. The shear stress (potential energy) is a linear (quadratic) function of $d$ in the elastic regime. The sets of configurations $i(d), f^{\langle 110\rangle}(d)$, and $f^{\langle 100\rangle}(d)$ are designed by thick solid lines in Fig. 2. From Figs. 2(a) and 2(b), the spontaneous GB migrations at $0 \mathrm{~K}$ are revealed by an energy drop and shear stress drop [from the configuration $i(0)$ to $f_{\langle 110\rangle}(0)$ at $d=0.314 \mathrm{~nm}$ ] or rise [from the configuration $f_{\langle 110\rangle}(0)$ to $i(0)$ at $-0.087 \mathrm{~nm}$ ]. Especially at $0 \mathrm{~K}$, the GB migrates from the configuration $i(d)$ at $d=0.314 \mathrm{~nm}$ to configuration $f^{\langle 110\rangle}(d)$ in agreement with the observation of mode $\langle 110\rangle$ at $0 \mathrm{~K}[10,23]$.

From Fig. 2, for a given $d$, in the range $-0.087 \mathrm{~nm}<d<$ $0.314 \mathrm{~nm}$ and at a finite temperature, a GB in a configuration $i(d)$ can migrate by thermal activation either to configurations $f^{\langle 110\rangle}(d)$ or $f^{\langle 100\rangle}(d)$. The nudge elastic band (NEB) method is applied to calculate the minimum energy path (MEP) between

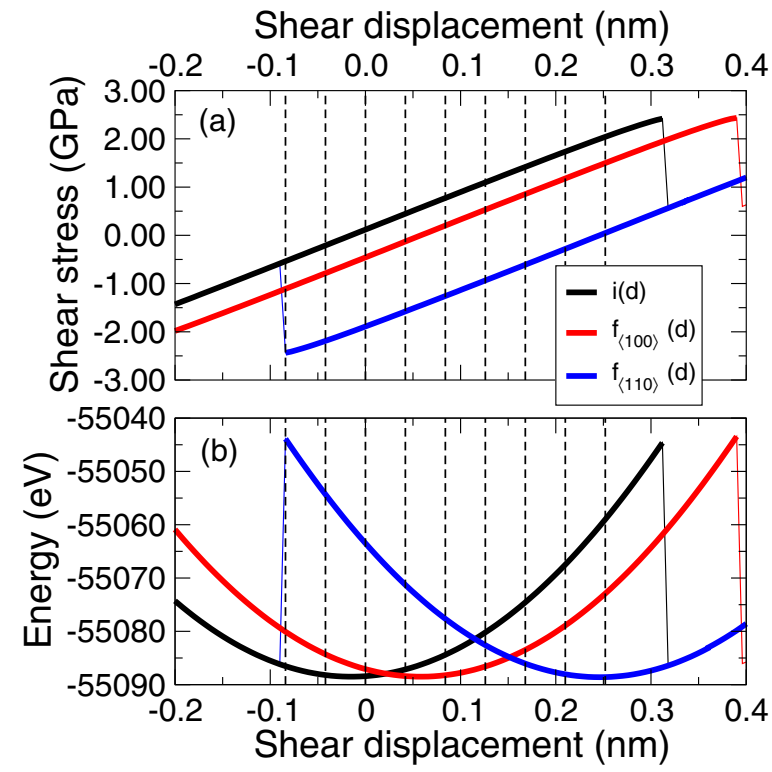

FIG. 2. (a) Shear stress and (b) energy of the bicrystal as a function of the relative shear displacement $d$ for different GB configurations calculated at $0 \mathrm{~K}$. NEB calculations have been performed for $d$ values mentioned by vertical dashed lines.

configurations $i(d)$ and $f^{\langle 110\rangle}(d)$ or $f^{\langle 100\rangle}(d)$ for values of $d$ (mentioned by vertical dashed lines in Fig. 2) ranging from $d=-0.084 \mathrm{~nm}$ to $d=0.252 \mathrm{~nm}$ following an arithmetic progression with a common difference of $0.042 \mathrm{~nm}$. A reaction coordinate (RC) is defined to indicate the GB migration progress: It is the cumulative distance (normalized by the total cumulative distance) between adjacent replicas in the configuration space (dimension $3 N, N$ the number of atoms). At $d=0.294 \mathrm{~nm}$, the MEP for the $\langle 110\rangle$ mode has been successfully calculated and will be thus reported in Fig. 8, but despite numerous efforts, the $\langle 100\rangle$ mode calculation did not converge.

\section{ELEMENTARY MECHANISMS OF THE GB MIGRATION MODES}

Below, we analyze the $\langle 110\rangle$ and $\langle 100\rangle$ modes GB migration MEPs for $d=0$, representative MEPs among calculated ones. Elementary mechanisms of the $\langle 110\rangle$ and $\langle 100\rangle$ modes are revealed. The occurrence of both $\langle 110\rangle$ and $\langle 100\rangle$ mode will be considered in Sec. IV through the computation of their activation energies as a function of the $\mathrm{SD} d$.

\section{A. Mode $\langle\mathbf{1 1 0}\rangle$}

The evaluation of the MEP following the $\langle 110\rangle$ mode has been performed using 80 NEB images in the NEB method. Judicious prebuilt intermediate configurations have been used to initialize NEB images in order to fasten the convergence (preliminary calculations using interpolate configurations between the initial and final configurations have been performed and report identical results). Intermediate configurations are designed from an appropriate mixing between the initial and final configurations. The choice of the simulation cell size along the $[\overline{1} 40]$ direction has been motivated by the high 


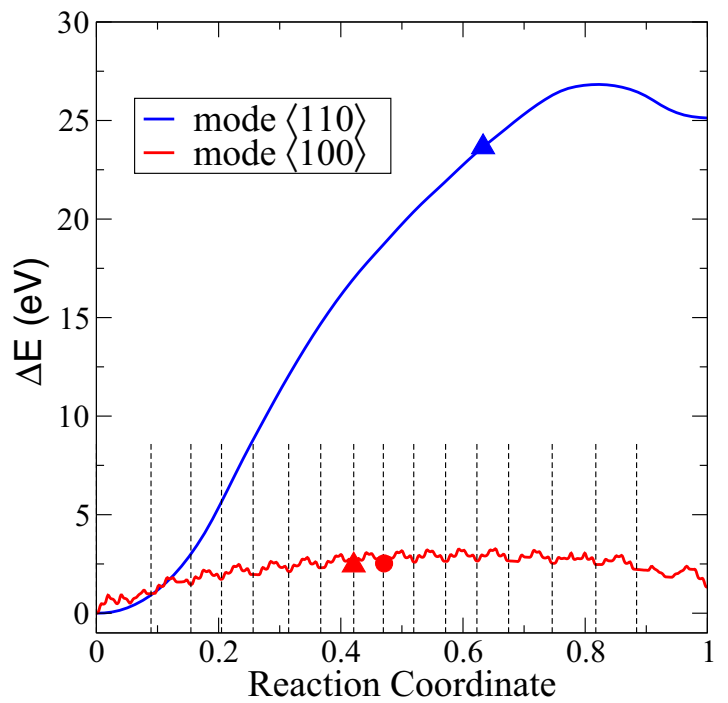

FIG. 3. MEP as a function of the RC for mode $\langle 110\rangle$ and $\langle 100\rangle$ at $d=0.0 \mathrm{~nm}$. Vertical dashed lines mention type I metastable states. Figures 4(a) and 4(b) report configurations corresponding to the blue $(R C=0.633)$ and red triangles $(R C=0.421)$. The red circle mentions the configuration at $R C=0.471$.

interaction energy between disconnections reported below and created along the $\langle 110\rangle$ mode. Especially, the MEPs following the $\langle 110\rangle$ mode calculated in smaller simulation cells $(5.35$ $\mathrm{nm}$ in the [1 $\overline{4} 0$ ] direction) do not display any disconnection nucleation but evidence a stick-slip relative motion of grain 1 compared to grain 2 (not shown).

Figure 3 reports the MEP between the configurations $i(0)$ and $f^{\langle 110\rangle}(0)$ as a function of the reaction coordinate (RC). The ordinate $\Delta E$ of Fig. 3 states for the difference between the total energy of the bicrystal along the MEP and its

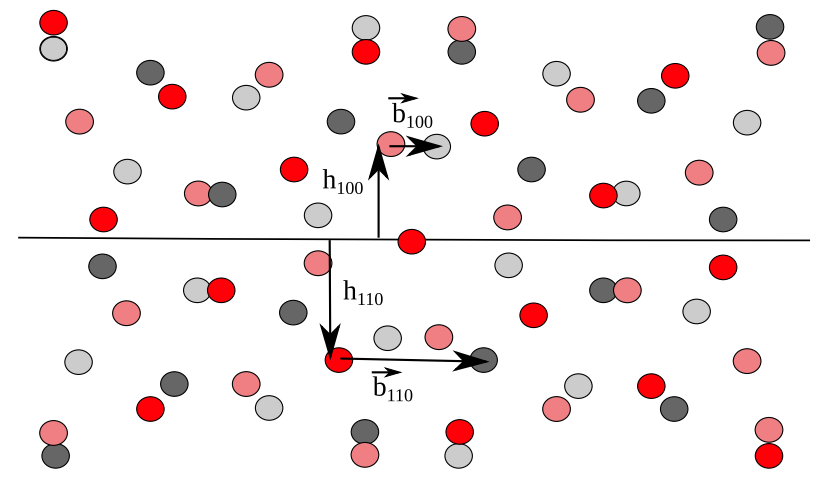

FIG. 5. Dichromatic pattern of the $\Sigma 17(410)$ grain boundary (GB). Same color code as Fig. 1(b). $h_{100}, h_{110}, \vec{b}_{100}$, and $\vec{b}_{110}$, respectively, report the disconnections heights and Burgers vectors for modes $\langle 100\rangle$ and $\langle 110\rangle$.

energy for $R C=0$. The MEP presents a maximum and no intermediate metastable state. Atomic configurations along the MEP evidence the nucleation and motion in opposite directions of two straight line GB steps, identified as disconnections. Figure 4(a) reports the atomic configuration for $R C=0.633$ (blue triangle in Fig. 3) displaying two disconnections. The Burgers vector of these disconnections is determined using a Burgers circuit analysis and is $\vec{b}_{110}=\frac{3}{17} a[1 \overline{4} 0](a=0.361 \mathrm{~nm}$ the $\mathrm{Cu}$ lattice parameter). Such Burgers vectors have a $0.263 \mathrm{~nm}$ norm corresponding to more than $2 / 3$ of the lattice parameter. This Burgers vector is reported on the dichromatic pattern Fig. 5 from which the coupling factor $\frac{h_{110}}{b_{110}}=-6 / 5$ ( $h_{110}$ is the algebraic step height) is deduced. This large Burgers vector induces highly distorted structural units beside the disconnections and likely a high elastic interaction energy between disconnections responsible for the absence of the metastable state in all $\langle 110\rangle$ mode calculated MEPs.

(a)

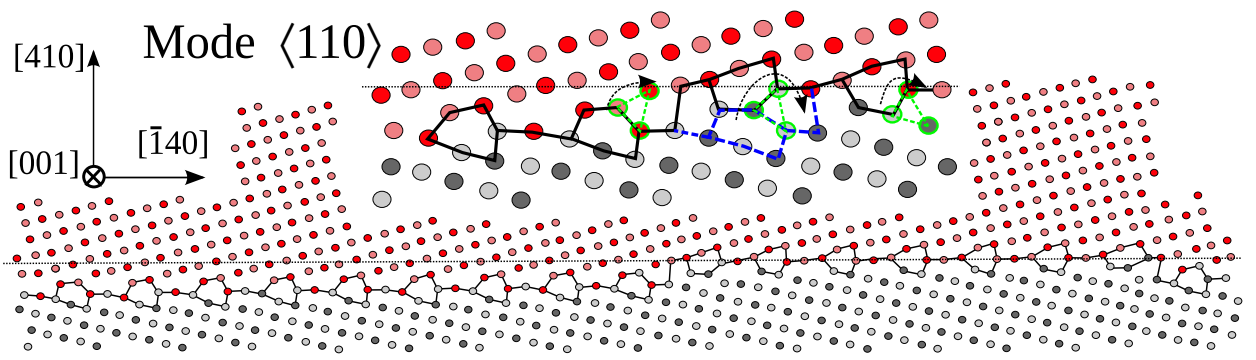

(b)

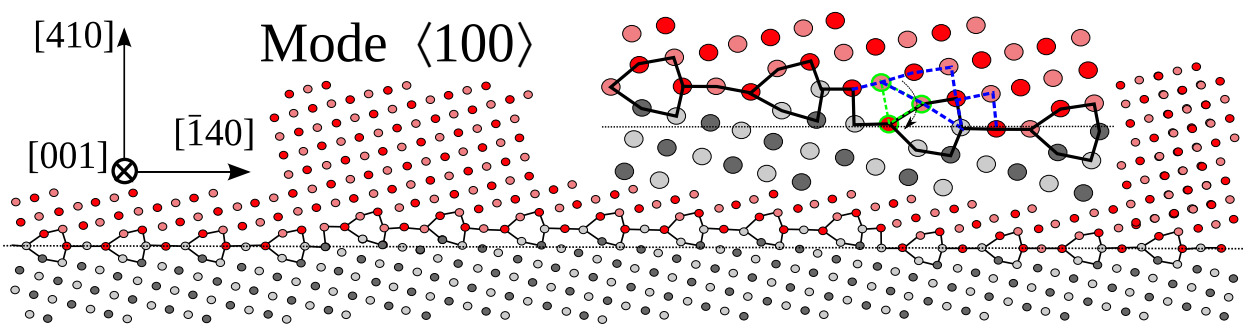

FIG. 4. Atomic configurations $(d=0)$ (a) along the $\langle 110\rangle$ mode MEPs for $R C=0.633$ (blue triangle in Fig. 3 ) and (b) the $\langle 100\rangle$ mode MEP for $R C=0.421$ (red triangle in Fig. 3). A horizontal line designs the initial position of the GB. The insets display a zoom on a disconnection and schematically report the main moving atoms (green circles). The black solid and blue dashed (in the insets) lines join atomic rows forming the structural units before and after the disconnection motion. 
Examining the configurations along the MEP, the motion of the disconnection essentially results from the coordinated displacement of atoms in a region extending over $1.5 \mathrm{~nm}$ around the disconnection. The rotation of three atomic rows [central green triangle in the inset of Fig. 4(a)] around the [001] axis induces the downward displacement of the structural unit and thus the motion of the disconnection. Besides, the structural units aside from the disconnection slightly distorts through the rotation (around the [001] axis) of two sets of three atomic rows [left and right green triangles in the inset of Fig. 4(a)]. Note that a careful analysis of the successive atomic configuration along the MEP does not show any atomic displacement along the [001] direction.

\section{B. Mode $\langle\mathbf{1 0 0}\rangle$}

The convergence of the NEB calculations for the MEP following the $\langle 100\rangle$ mode is computationally demanding. For each SD $d$, the overall MEP results from about a dozen NEB simulations involving 60 to 80 NEB images performed between prebuilt intermediate metastable configurations used as initial and final states in the NEB method. These intermediate configurations are designed from an appropriate mixing between the initial and final configurations and are similar to the type $A$ metastable state (TAMS) described below. For each of these NEB calculations, NEB images are initialized with interpolate configurations between the initial and final states.

Figure 3 reports the MEP between the configurations $i(0)$ and $f^{\langle 100\rangle}(0)$ as a function of the reaction coordinate (RC) and involves an overall total of 800 NEB images. Examining atomic configurations along the MEP, the $\langle 100\rangle$ mode GB migration occurs through the nucleation and the motion of two disconnections. In addition, the MEP shows numerous metastable states that can be classified in two types: the type $A$ metastable states (TAMS) (vertical dashed lines in Fig. 3) present two straight line disconnections (along the [001] direction); the type $B$ metastable states (TBMS) present some kinks along the disconnections lines. We below first describe the straight line disconnection (TAMS) and their migration mechanism as seen along the [001] axes and then the disconnection kinks (TBMS) structure and migrations.

Figure 4(b) reports the configuration $(R C=0.421)$ of a TAMS displaying two straight line disconnections. Their Burgers vectors are $\vec{b}_{100}=\frac{1}{17} a[1 \overline{4} 0]$ and are reported in the dichromatic pattern Fig. 5. The GB coupling factor is $\frac{h_{100}}{b_{100}}=1 / 2$ ( $h_{100}$ the algebraic step height). The disconnection motion as seen along the [001] axes and driving the system from the TAMS $R C=0.421$ (red triangle) to the next TAMS $R C=0.471$ (red circle in Fig. 3) mainly involves moving atoms in the close proximity of the disconnection: The rotation (around the [001] axis) of three atomic rows [green triangle in the inset of Fig. 4(b)] close to the disconnection causes the upward displacement of the structural unit. Moreover, this rotation is accompanied by the displacement of atoms of the right green triangle vertices along the [001] direction by a half lattice parameter.

From the examination of the TBMS configurations along the MEP, up to four disconnection kinks along one single disconnection are observed: The simulation cell along the

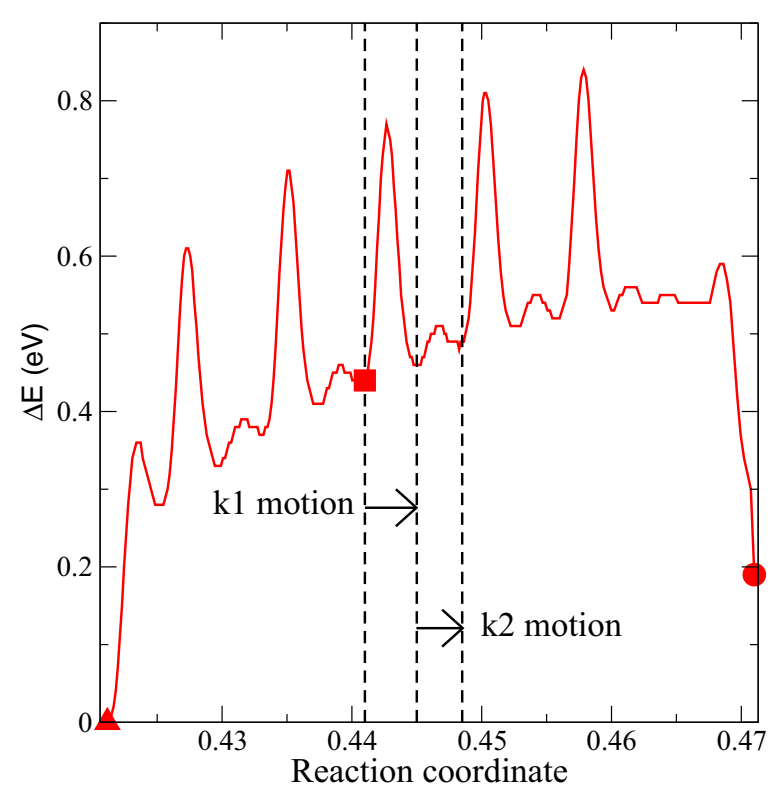

FIG. 6. MEP as a function of the RC for mode $\langle 100\rangle$ at $d=$ $0.0 \mathrm{~nm}$ between $R C=0.421$ and $R C=0.471$ in a simulation cell with $z$ size $=4.2 \mathrm{~nm}$. Vertical dashed lines mention $R C=0.441$, 0.445 , and $R C=0.445$. The red square mentions the configuration at $R C=0.441$.

[001] (z-axis) direction is small $(1.4 \mathrm{~nm})$ and induces a spurious interaction between the kinks and their periodic images. Hence, additional NEB calculations using a $4.2 \mathrm{~nm}$ simulation cell along the [001] direction between the two TAMS $R C=0.421$ and $R C=0.471$ (red triangle and circle in Fig. 3) have been performed. The simulation cell contains 47040 atoms. 300 NEB images are used in the NEB method. Since preliminary results (with a smaller number of NEB images) using interpolate configurations between the initial and final states to initialize NEB images have evidenced disconnection kinks and in order to fasten the convergence of the NEB method, some intermediate configurations displaying the presence and the motion in the opposite direction of the disconnection kinks are created and used to initialize NEB images. The intermediate configurations are designed from a judicious mixing between the configurations at $R C=0.421$ and $R C=0.471$. The use of similar prebuilt configurations in the small simulation cell ( $z$ size $1.4 \mathrm{~nm}$ ) between $R C=0.421$ and $R C=0.471$ provides identical results to those reported in Fig. 3.

Figure 6 reports the accurate NEB calculations performed between two TAMS $R C=0.421$ and $R C=0.471$ (red triangle and circle of Fig. 3 ) in the large simulation cell. The ordinate $\Delta E$ of Fig. 6 states for the difference between the total energy of the bicrystal along the MEP and its energy for $R C=0.421$.

The disconnection motion occurs through the nucleation of two disconnection kinks and their further motions in the opposite [001] direction. The nucleation energy $E_{\text {nucleation }}^{\text {kink }}=$ $0.30 \mathrm{eV}$ of two disconnection kinks is determined from Fig. 6 as in Ref. [24].

Figure 7(a) displays the atomic configuration for $d=0$ at $R C=0.441$ (red square in Fig. 6) in the large simulation cell 
(a)

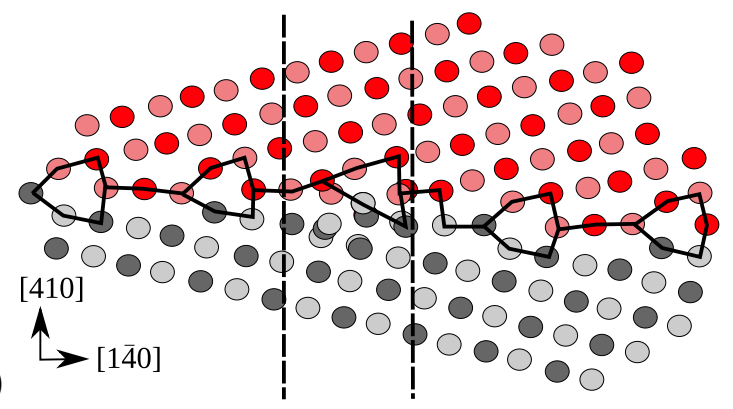

(b)

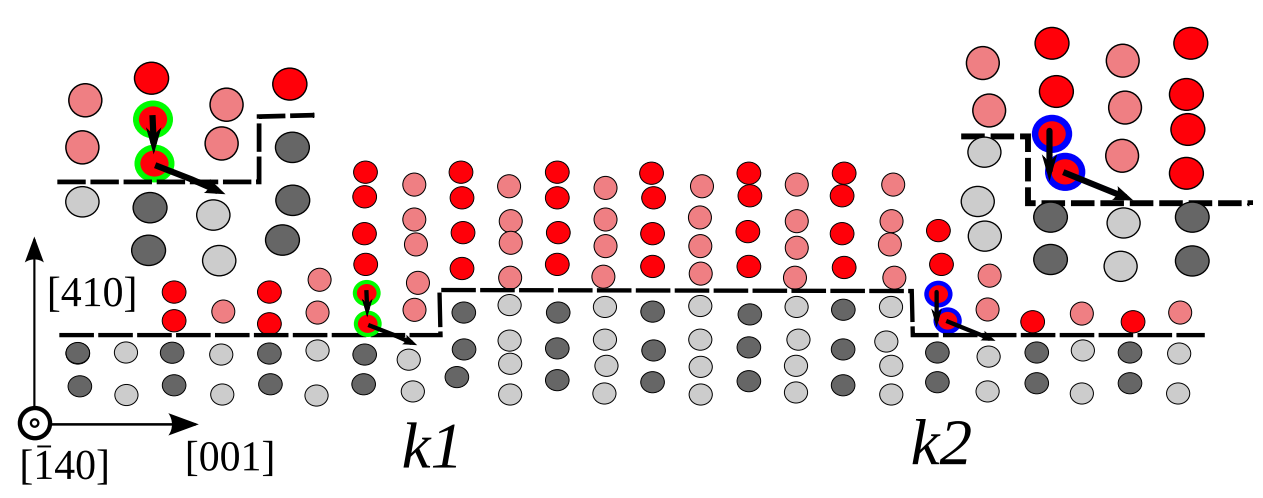

FIG. 7. (a) Atomic configurations along the $\langle 100\rangle$ mode MEP for $d=0$ and for $R C=0.441$ projected along the [001] direction and (b) projected along the [140] direction where only atoms between the two vertical dashed lines of (a) are displayed. Left and right insets present details of the disconnections kinks $k_{1}$ and $k_{2}$. The simulation $z$ size is $4.2 \mathrm{~nm}$. A dashed line mentions the position of the GB. Same color code as Fig. 1(b).

projected along the [001] direction. Figure 7(b) displays the same configuration projected along the [1]40] direction where only atoms between the two vertical dashed lines of Fig. 7(a) are displayed. Figure 7(b) displays two disconnection kinks $k 1$ and $k 2$ and the mechanism of their motion in the large simulation cell.

Analyzing Fig. 6, the migration energy barriers of the $k 1$ (from $R C=0.441$ to 0.445 ) and $k 2$ (from $R C=0.445$ to 0.448 ) kinks are $0.31 \mathrm{eV}$ and $0.04 \mathrm{eV}$. Both $k 1$ and $k 2$ kinks motions are mainly induced by a similar displacement of two atoms (respectively, green and blue circled atoms in Fig. 7 and insets) close to the disconnection kinks. Especially, the lower atoms shift by half a lattice parameter in the [001] direction, in agreement with observations reported above on the migration mechanisms of TAMS. The opposite directions of these displacements with respect to the disconnection kink orientations explain the difference in their activation energies.

Note however that since the NEB method is supposed to provide the minimum energy path and since the $k 2$ migration energy barrier is almost eight times smaller than the $k 1$ one, one would expect the NEB method to provide a MEP involving only $k 2$ kink motions (energetically cheaper than $k 1$ motions). However, the results of Fig. 6 involve both $k 1$ and $k 2$ motion: Actually, the prebuilt intermediate states used to initialize the NEB images that involve the presence and the motion in the opposite direction of disconnection kinks influence the convergence of the NEB method towards a "metastable" energy path. Results of Fig. 6 remain, however, highly relevant to evidence the $k 1$ and $k 2$ kink motions.

\section{COMPETITION BETWEEN THE $\langle 110\rangle$ AND $\langle 100\rangle$ MODES}

To compare the $\langle 110\rangle$ and $\langle 100\rangle$ modes, the GB migration activation energies $E_{\langle 110\rangle}^{\text {act }}$ and $E_{\langle 100\rangle}^{\text {act }}$, defined as the difference between the highest reached energies along the MEP and the initial one $(R C=0)$, are computed as a function of the $\mathrm{SD}$ in the small simulation cell $(z$ size $=1.4 \mathrm{~nm})$. Calculations in the large simulation cell require heavy computational efforts reasonably not generalizable to all calculated MEPs. Although, since the disconnection nucleation dominates the GB migration activation energy, our calculations give a relevant picture of the modes competition. The activation energies $E_{\langle 110\rangle}^{\text {act }}$ and $E_{\langle 100\rangle}^{\text {act }}$ reported in Fig. 8 are decreasing functions of the SD $d$ : an Akima spline interpolation is used between and beyond the calculated points [25]. For $d \lesssim 0.28 \mathrm{~nm}(\gtrsim)$, the $\langle 100\rangle$ mode activation energy is smaller (higher) than the $\langle 110\rangle$ mode's one. The cancellation of the activation energy $E_{\langle 110\rangle}^{\text {act }}$ as interpolated occurs for $d=0.318 \mathrm{~nm}$ in agreement with the $\langle 110\rangle$ mode GB migration at $0 \mathrm{~K}$ at $d=0.314 \mathrm{~nm}$ (Fig. 2).

The probabilities per unit time that the GB migrates following the $\langle 110\rangle$ and $\langle 100\rangle$ are a function of the activation energies:

$$
\begin{gathered}
v_{\langle 110\rangle}=v_{0} e^{-\frac{E_{[110\rangle}^{\mathrm{act}}}{k T}} \\
v_{\langle 100\rangle}=v_{0}^{\prime} e^{-\frac{E_{|100\rangle}^{\mathrm{act}}}{k T}},
\end{gathered}
$$

where $v_{0}$ and $v_{0}^{\prime}$ are roughly the Debye frequency. Hence, in a prefect bicrystal, increasingly shearing the GB at low 


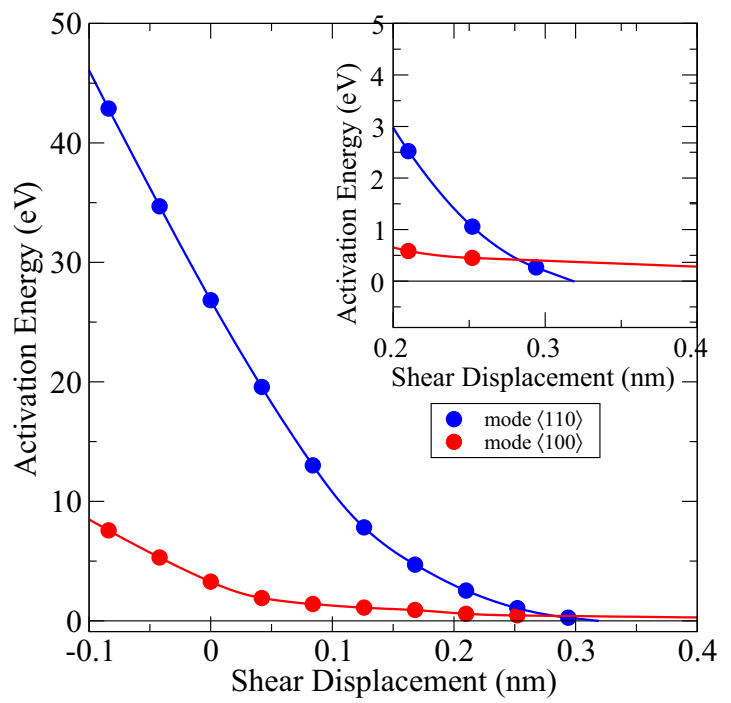

FIG. 8. Activation energies (point) of $\langle 110\rangle$ and $\langle 100\rangle$ GB migration modes as a function of the SD $d$. Solid lines are Akima splines interpolations of these curves. Inset: zoom of the curves in the $0.2-0.4 \mathrm{~nm}$ shear-displacement range.

temperature $\left[k T \ll E_{\langle 100\rangle}^{\text {act }}(d=0)\right.$, the shear displacement and stress increase until a sufficient decrease of the activation energies to allow a GB migration on the experiment timescale. At $T=0$, the GB migrates following the $\langle 110\rangle$ mode, which first cancels its activation energy as a function of $d$ as shown in Fig. 8. At high temperatures, GB migration probabilities for both modes are higher, and the GB can quickly migrate for small $\mathrm{SD}(d \ll 0.318 \mathrm{~nm})$. However, since $E_{\langle 100\rangle}^{\mathrm{act}} E_{\langle 110\rangle}^{\text {act }}$ for $d \ll 0.318 \mathrm{~nm}$, the mode $\langle 100\rangle$ has a larger probability to occur. Between these two regimes, at intermediate temperatures, both GB migration modes can jointly occur if the GB migrates for SD close to the activation energies crossing $(d \simeq$ $0.28 \mathrm{~nm}$ ). These predictions agree with MD simulations of GB migrations at constant shear velocity in model bicrystals [10].

In a realistic polycrystalline material, the homogeneous disconnection nucleation as studied from perfect bicrystals in this study may presumably play a role, but one also expects the inhomogeneous nucleation of disconnections at triple lines [26] or from the interactions between bulk dislocations and GB [3]. Therefore, in addition to the GB migration activation energies, the disconnection kink nucleation energy barriers constitute relevant data for experiments providing noticeably the temperature dependence of the disconnection mobility. Though we are not aware of disconnection mobility measurements, observations of disconnections motions have already been reported [27].

\section{CONCLUSION}

Both $\langle 110\rangle$ and $\langle 100\rangle$ GB migration modes of the $\Sigma 17(410)$ GB in a copper bicrystal have been theoretically studied. For both modes, GB migration occurs through the nucleation and motion of disconnections, suggesting that this mechanism, already evidenced for a $\Sigma 13(320)$ GB [24], is general in shear-coupled GB migration. However, the detailed GB migration mechanisms can significantly vary: For the $\Sigma 17(410) \mathrm{GB}$, whereas the $\langle 110\rangle$ mode has no metastable state, the $\langle 100\rangle$ mode MEP presents multiple metastable states, some of them evidencing some kinks along the disconnection lines. The presence of metastable states has been presumably related to the norm of the disconnection Burgers vector. Examining the $\langle 100\rangle$ mode, the kink disconnection nucleation and migration energies have been evaluated providing valuable data for experiments, in which inhomogeneous disconnections nucleation is expected. Besides, investigating the competition between both GB modes through homogeneous disconnections nucleation, the activation energies of the $\langle 100\rangle$ mode have been shown to be smaller than those of the $\langle 110\rangle$ one except for very high stresses $(d>0.28 \mathrm{~nm}$ in our case), in agreement with MD simulations on model bicrystal. These results significantly improve our knowledge on the GB migrations mechanisms and on the conditions under which they occur. To go beyond, getting closer to real materials, the present work needs to be extended to study the migration of asymmetric GB and the inhomogeneous nucleation of disconnections at triple lines in polycrystals [26] or from the interaction between dislocations and GB.

\section{ACKNOWLEDGMENTS}

This work was performed using HPC resources from CALMIP (Grant No. 2012-12172). N.C. acknowledges J. Morillo and M. Benoit for useful discussions.
[1] M. Jin, A. Minor, E. Stach, and J. Morris, Acta Mater. 52, 5381 (2004).

[2] M. Legros, D. Gianola, and K. Hemker, Acta Mater. 56, 3380 (2008).

[3] A. Rajabzadeh, M. Legros, N. Combe, F. Mompiou, and D. Molodov, Philos. Mag. 93, 1299 (2013).

[4] A. Rajabzadeh, F. Mompiou, S. Lartigue-Korinek, N. Combe, M. Legros, and D. Molodov, Acta Mater. 77, 223 (2014).

[5] Q. Yu, M. Legros, and A. Minor, MRS Bull. 40, 62 (2015).

[6] J. Schäfer and K. Albe, Acta Mater. 60, 6076 (2012).

[7] T. Gorkaya, D. A. Molodov, and G. Gottstein, Acta Mater. 57, 5396 (2009).
[8] D. A. Molodov, T. Gorkaya, and G. Gottstein, Scr. Mater. 65, 990 (2011).

[9] G. Gottstein, D. Molodov, L. Shvindlerman, D. Srolovitz, and M. Winning, Curr. Opin. Solid State Mater. Sci. 5, 9 (2001).

[10] J. W. Cahn, Y. Mishin, and A. Suzuki, Acta Mater. 54, 4953 (2006).

[11] L. Wan and S. Wang, Phys. Rev. B 82, 214112 (2010).

[12] M. Velasco, H. V. Swygenhoven, and C. Brandl, Scr. Mater. 65, 151 (2011).

[13] H. Khater, A. Serra, R. Pond, and J. Hirth, Acta Mater. 60, 2007 (2012).

[14] E. R. Homer, S. M. Foiles, E. A. Holm, and D. L. Olmsted, Acta Mater. 61, 1048 (2013). 
[15] C. Brandl, T. C. Germann, A. G. Perez-Bergquist, and E. K. Cerreta, Mater. Res. Lett. 1, 220 (2013).

[16] C. P. Race, J. von Pezold, and J. Neugebauer, Phys. Rev. B 89, 214110 (2014).

[17] W. T. Read and W. Shockley, Phys. Rev. 78, 275 (1950).

[18] J. W. Cahn, Y. Mishin, and A. Suzuki, Philos. Mag. 86, 3965 (2006).

[19] Note that a sliding mechanism, not investigated in this paper, is also reported at high temperature (around 1000-1200 K) for both low and high angle GBs [10].

[20] S. J. Plimpton, J. Comput. Phys. 117, 1 (1995).
[21] Y. Mishin, D. Farkas, M. J. Mehl, and D. A. Papaconstantopoulos, Phys. Rev. B 59, 3393 (1999).

[22] A. P. Thompson, S. J. Plimpton, and W. Mattson, J. Chem. Phys. 131, 154107 (2009).

[23] Y. Mishin, M. Asta, and J. Li, Acta Mater. 58, 1117 (2010).

[24] A. Rajabzadeh, F. Mompiou, M. Legros, and N. Combe, Phys. Rev. Lett. 110, 265507 (2013).

[25] H. Akima, J. ACM 17, 589 (1970).

[26] M. Aramfard and C. Deng, Modelling Simul. Mater. Sci. Eng. 22, 055012 (2014).

[27] F. Mompiou, M. Legros, A. Boé, M. Coulombier, J. Raskin, and T. Pardoen, Acta Mater. 61, 205 (2013). 